\title{
VIII. Politische Irritationen und Komplikationen
}

Die Istanbuler Botschafterkonferenz vom April 1956 und die Anwendung der Hallsteindoktrin im Nahen Osten schufen im israelisch-arabisch-deutschen Dreieck eine neue Situation. Bonn nahm die Veränderungen rasch wahr, die Araber folgten bald nach, und nur die Israelis verweilten weiter im Narrenparadies. Nicht alle israelischen Diplomaten verkannten die neuen Verhältnisse, obwohl Shinnar das israelische Außenministerium weiter mit irreführenden Erklärungen aus dem Auswärtigen Amt versorgte. Shinnars grenzenloser Optimismus und sein uneingeschränktes Vertrauen in die Ernsthaftigkeit und Aufrichtigkeit der deutschen Partner auf diplomatischer und politischer Ebene trübten den Blick der israelischen Regierung und schufen ständig neue Illusionen.

Auf der Botschafterkonferenz wurde, wie erwähnt, beschlossen, „zu gegebener Zeit" Beziehungen mit Israel aufzunehmen. Mindestens ein Teil der westdeutschen Diplomaten in arabischen Hauptstädten wußte, was das bedeutete: eine unbefristete Verschiebung. Diese Diplomaten erlebten den $\mathrm{Haß}$ gegen den Staat Israel im täglichen Kontakt mit ihrer Umwelt und vertraten die Auffassung, daß kein Fortschritt möglich sei, solange dieser $\mathrm{Haß}$ anhielt. Bestimmte Kreise in Bonn, besonders im Auswärtigen Amt, erkannten diesen Sachverhalt und vertrösteten Shinnar, Goldmann und andere jüdische Vertreter wiederholt auf den "geeigneten Zeitpunkt", der jedoch so schnell nicht eintraf. Zuerst lagen die Bundestagswahlen dazwischen, dann wurde die Aufnahme von Beziehungen durch die Wahlen in Israel verhindert, und später kamen militärische Spannungen bzw. jeweils eine die beiden Seiten belastende diplomatische Begebenheit dazwischen oder es wurden "entscheidende westliche Interessen im Nahen Osten" vorgeschoben, die es zu verteidigen galt.

Opposition gegen die Aufnahme diplomatischer Beziehungen zwischen der Bundesrepublik und Israel gab es auch von alliierter Seite. Die Bundesregierung ließ sich also Zeit mit der Wahl des "geeigneten Zeitpunkts“. Es dauerte eine Weile, bis die Israelis das Spiel durchschauten und begannen, Druck auf Bonn auszuüben. Von Gewissensbissen geplagt, suchte ein Teil des politischen Establishments der Bundesrepublik nach Möglichkeiten der Entschädigung für Israel. Während das Auswärtige Amt im folgenden Jahrzehnt alles in seiner Macht stehende tat, um die Annäherung zwischen beiden Staaten zu verhindern, bemühten sich einige Politiker in Bonn, dem jungen Staat wenigstens hinsichtlich seiner dringendsten Bedürfnisse unter die Arme zu greifen.

\section{Die Suezkrise}

Die israelische Regierung war zunächst streng darauf bedacht, eine Konfrontation mit der Bundesrepublik oder eine Krise zwischen beiden Staaten zu vermeiden. 
Der Stab der Israel-Mission und die anderen diplomatischen Vertretungen des jüdischen Staates im Ausland erhielten Anweisung, an der gewöhnlichen Arbeitsroutine festzuhalten. Als Ziel der israelischen Diplomatie galt bis Mitte 1956 die Aufnahme diplomatischer Beziehungen zur Bundesrepublik Deutschland und die Einrichtung einer westdeutschen Mission in Israel. Dies änderte sich jedoch, als Moshe Sharett im Amt des Außenministers Ende Juni von Golda Meir abgelöst wurde. Die neue Außenministerin Meir war doktrinärer als ihr Vorgänger und ihre Abneigung gegenüber Deutschland stärker ausgeprägt. Unter ihrer Führung ließ der Druck der israelischen Außenpolitik auf Bonn, die gegenseitige Beziehungen allmählich enger zu gestalten, merklich nach. Israel forderte nun den vollen diplomatischen Austausch auf Botschafterstufe und war nicht mehr bereit, über irgendwelche $Z$ wischenlösungen zu verhandeln. Dies ist nicht nur als Reaktion auf die in Israel als Affront empfundene deutsche Verzögerungstaktik zu werten. Auch Meirs Hartnäckigkeit und vielleicht auch Gleichgültigkeit gegenüber Deutschland dürften dabei eine Rolle gespielt haben. Golda Meir war nicht an minimalen Lösungen interessiert. Alles oder nichts lautete die Devise. Meir ordnete an, „aufzuhören, in Bonn um Beziehungen zu betteln“. 1 Ihre Handschrift machte sich in der internen Korrespondenz und im Verhalten der israelischen Diplomaten erst allmählich bemerkbar. Golda Meir stellte klar, daß die Initiative zur Ausgestaltung der gegenseitigen Beziehungen von der Bundesrepublik ausgehen müsse. Sie hielt damit am traditionellen israelischen Standpunkt fest, der im Laufe der Jahre allerdings immer großzügiger ausgelegt worden war.

Die Außenministerin arbeitete auch in dieser Frage eng mit dem Ministerpräsidenten zusammen, trotz unterschiedlicher Haltungen. Der doktrinären, Deutschland äußerst abgeneigten Meir stand ein pragmatischer, möglicherweise auch versöhnlicher Ben Gurion gegenüber. Die Holocaust-Erinnerung belastete ihn zwar ebenso, wie seine häufigen Äußerungen zu diesem Thema und die Verwandlung des Eichmannprozesses in ein Lehrstück für die Nation zeigen, doch er weigerte sich, die Deutschen mit dem Etikett der Kollektivschuld zu versehen. Statt dessen pflegte er die Bedeutung von Sühne und Eigenverantwortung zu betonen. ${ }^{2}$ Mit besonderer Vorliebe benutzte er den Begriff „Neues Deutschland“, womit er Adenauers Deutschland meinte, ein Land, das, so Ben Gurion, dem Nationalsozialismus und dem Antisemitismus den Rücken gekehrt habe und bereit sei, die Opfer der NS-Gewaltherrschaft zu entschädigen. Um diesen fundamentalen Wandel zu veranschaulichen, wies er auf die Schilumim und andere Formen der Wiedergutmachung hin. Ostdeutschland dagegen, das sich weigerte, Entschädigung zu leisten, blieb in seinen Augen ein „Schurkenstaat“. Ganz im Gegensatz dazu drückte Meir der ganzen deutschen Nation, in West und Ost, das Kainsmal auf. Die Außenministerin zeigte wenig Verständnis für Nuancen oder Versöhnung. Ben Gurion dagegen war dankbar für jede Hilfe aus Deutschland, und Meir

1 Gideon Rafael an den Außenminister, handschriftliche Ergänzung von Golda Meir vom 26. 1. 1960, ISA, 3309/13.

2 Ben Gurion an Adenauer vom 1. 1. 1961, ISA, 3533/12; Protokoll über das Interview der Herausgeber von Ma'ariv (Tel Aviv) mit Ben Gurion vom 13. 9. 1960, BGA, Protocols' file. 
fügte sich Ben Gurions Politik letztlich nur mit Rücksicht auf die akuten Probleme des jungen Staates.

Meirs emotionale Schwierigkeit, die neue Realität zu akzeptieren, zeigte sich besonders deutlich in der Diskussion über die Aufnahme diplomatischer Beziehungen mit der Bundesrepublik. Vorwürfe in der Knesset hinsichtlich ihrer mangeinden Bereitschaft zur Aufnahme solcher Beziehungen wies sie trotz - zuvor dargelegter - Anzeichen einer gegenteiligen Haltung zurück. ${ }^{3}$ Sie zog es vor, die Fakten zu verschleiern. Das ungeliebte politische Ziel verfolgte sie nur zögerlich.

Bonn mußte dagegen schon bald Farbe bekennen: Im Juni 1956 endete die britische Herrschaft über den Suezkanal, und Oberst Nasser wollte den Lebensstandard seines Volkes durch den Bau des Assuan-Staudammes verbessern. Dazu hielt er sowohl im Westen als auch im Osten nach Kapital Ausschau. Die Bundesrepublik erklärte sich zur Mitwirkung am Projekt bereit, doch sie war aus wirtschaftlichen Gründen nicht in der Lage, es allein tragen. Die USA, die Weltbank und Großbritannien lehnten eine Teilnahme aus politischen Gründen ab, und ob die Sowjetunion fähig sein würde, ein solches Projekt selbständig durchzuführen, wurde bezweifelt. Ägypten sah sich also gezwungen, den Bau aus eigenen Mitteln zu finanzieren. Nasser zögerte nicht lange, und am 26. Juli 1956 verstaatlichte er die Suezkanalgesellschaft, aus deren Einnahmen er die Errichtung des Staudammes zu finanzieren hoffte. Die Verstaatlichung richtete sich direkt gegen französische und britische Interessen, Nachteile für den Schiffverkehr wurden ebenfalls befürchtet. Die negativen Folgen für die israelische Schiffahrt und den israelischen Handel ließen in der Tat nicht lange auf sich warten. Unter dem Vorwand des Kriegszustandes mit Israel blockierte Ägypten die Durchfahrt für die israelische Handelsflotte und für Schiffe anderer Länder mit Gütern von und nach Israel. Dreimal trafen die Kanalbenutzer zu Beratungen zusammen, jeweils ohne israelische Beteiligung. Die israelische Regierung appellierte an verschiedene Teilnehmer, auch an die Bundesrepublik, ihre Schiffahrtsinteressen zu verteidigen. Sie erhielt jeweils eine freundliche, aber ausweichende Antwort.

Das Jahr 1956 sollte für Israel besonders schwer werden. Die Terrorangriffe mehrten sich, der Suezkanal und der Golf von Akaba bzw. der Zugang zum indischen und zum pazifischen Ozean wurden für israelische Schiffe gesperrt, und Ägypten häufte große Mengen von Waffen an, so daß sich Israel zu einem Präventivschlag veranlaßt fühlte. ${ }^{4}$ Ben Gurion förderte die militärische und politische Zusammenarbeit mit Frankreich, das damals in den von Ägypten unterstützten algerischen Unabhängigkeitskrieg verwickelt war. Die Franzosen lieferten den Israelis dringend benötigtes Rüstungsmaterial, darunter Kampfflugzeuge und Panzer. Über das französische Militär entwickelten sich im Laufe der Zeit direkte Kanäle zwischen Israel und der Bundeswehr bzw. dem Bundesnachrichtendienst. Zudem sicherte sich Israel durch die enge Zusammenarbeit mit Frankreich Zugang zu deutschem Know-how und Rüstungsgütern aus deutscher Produktion.

3 KNeSSET-PROTOKOLle [Original hebr.], 3. Knesset, 146. Sitzung am 2. 7. 1956, S. 21552156.

4 Herzog, The Suez-Sinai-Campaign, S. 17-53. 
Von der internationalen Solidarität enttäuscht, erwogen Großbritannien und Frankreich militärische Schritte zur Wahrung ihrer Interessen am Suezkanal und fanden in Israel einen willigen Partner für eine gemeinsame Operation gegen Ägypten. Israel ließ sich in einen klassischen Kolonialkrieg verwickeln, der in erster Linie den militärischen und materiellen Interessen Großbritanniens und Frankreichs diente. Andererseits hoffte man in Jerusalem, damit die Beziehungen zu den beiden Großmächten vertiefen, dem wichtigsten arabischen Feind eine vernichtende Niederlage zufügen, die innere Sicherheit wiederherstellen, die Seeblockaden durchbrechen und neues Territorium hinzugewinnen zu können. Am 29. Oktober 1956 drangen israelische Fallschirmspringer in der Sinaiwüste tief auf ägyptisches Gebiet vor und eröffneten damit den Krieg. Britische und französische Truppen zogen rasch nach. Aus einer begrenzten Operation zur Wahrung kolonialer Interessen wurde bald ein größerer diplomatischer Eklat mit weltweiten Konsequenzen, der an den Rand einer nuklearen Katastrophe führte. Die USA und die Sowjetunion stellten sich auf Ägyptens Seite und gegen die drei Verbündeten des Suezfeldzugs. Kurz darauf erfolgte die blutige und propagandistisch fatale Unterdrückung des ungarischen Aufstands durch die Sowjetunion. Der gleichzeitige Ausbruch der beiden Krisen kam Moskau höchst ungelegen und zwang den Kreml zu einer extremen Haltung in beiden Fragen. Im Gegensatz dazu konnte sich das Weiße Haus einen flexibleren Standpunkt zum ungarischen Volksaufstand leisten, während es im Nahen Osten sämtliche politischen und diplomatischen Mittel mobilisierte, um die Kriegsgegner Ägyptens zum Rückzug zu zwingen.

In der Bundesrepublik wurden die Ereignisse in Ungarn mit großer Sorge verfolgt und als weiteres Anzeichen brutaler sowjetischer Machtpolitik gewertet. Die Aussichten auf eine baldige Wiedervereinigung schienen weiter $\mathrm{zu}$ schwinden. Von der Suezkrise fühlte man sich dagegen weniger betroffen. ${ }^{5}$ Sie wurde nicht als unmittelbare Gefahr, sondern höchstens als ferne Bedrohung einer nuklearen Konfrontation empfunden, für die man die antiägyptische Koalition verantwortlich machte.

Die westdeutsche Öffentlichkeit hatte für die Militäroperation gegen Ägypten wenig Verständnis. Adenauer mußte nun Führung beweisen. Große Teile der Öffentlichkeit und der Presse, die SPD, zahlreiche Vertreter der CDU/CSU und das Auswärtige Amt verurteilten die Operation, wenn auch die Meinungen über die israelische Rolle geteilt waren. Während die Motive des jüdischen Staates teilweise auf Verständnis stießen, erwies sich die Kooperation mit „imperialistischen Mächten" als Belastung für Israels Ansehen. Das Auswärtige Amt war zu vollständiger Kooperation mit dem amerikanischen State Department gegen Israel bereit. Der westdeutschen Diplomatie bot die jüngste Nahostkrise eine willkommene Gelegenheit zur Bekundung der Solidarität mit den USA und zur Disziplinierung Israels. Kritische Stimmen in Bonn hatten sich schon bei früheren Gelegenheiten, während der Zwischenfälle an Israels Grenzen und den verschiedenen

5 BARING, Sehr verehrter, S. 199; Von Brentano an Adenauer vom 31. 10. 1956, BArch, N 1239/156 Nr. 303 und 304; Sir F. Hoyer-Millar an das Außenministerium vom 8.11. 1956, PRO, FO 371/124513. 
Verurteilungen des jüdischen Staates im UNO-Sicherheitsrat, gemeldet. Nun erwog das Auswärtige Amt Sanktionen gegen Israel, darunter die Einstellung der Schilumimlieferungen, um den amerikanischen Forderungen gegenüber Israel Nachdruck zu verleihen. ${ }^{6}$ Die amerikanische Regierung drohte Israel mit einer Wirtschaftsblockade, falls ihre "Ratschläge“ nicht befolgt würden, und rief die Bundesregierung auf, sich ihrer Politik anzuschließen. ${ }^{7}$ Unklar bleibt, wie weit das amerikanische Außenministerium mit seiner Forderung an die Bundesrepublik hinsichtlich der Einstellung der Schilumimlieferungen ging und ob das Auswärtige Amt einen solchen Schritt aus eigener Initiative anbot.

Bundeskanzler Konrad Adenauer markierte den europäischen Staatsmann: Angesichts der sowjetischen Bedrohung und der amerikanischen Vormachtstellung hielt er es für die Pflicht der Bundesrepublik, sich zu Europa zu bekennen. Er war darüber hinaus von der Notwendigkeit einer harten Haltung gegenüber dem Kommunismus und dem sowjetischen Expansionsdrang überzeugt. Der Kanzler warf den Amerikanern in diesem Zusammenhang Gleichgültigkeit gegenüber dem Nahen Osten vor. Würden die USA den Assuan-Staudamm finanzieren, so seine Meinung, wären die ägyptischen Massen beschäftigt und würden sich nicht auf Abenteuer einlassen. Statt dessen fördere die amerikanische Politik den aggressiven arabischen Nationalismus. ${ }^{8}$ Der Kanzler schätzte die Politik von Eisenhower und Dulles in diesem Konflikt nicht, trotz seines freundschaftlichen Verhältnisses zu beiden Politikern. Als Europäer stand er der amerikanischen Haltung, die er als überheblich empfand, kritisch gegenüber. Unabhängig davon nannte er Nasser in internen Gesprächen einen „Gangster" und hatte keine Bedenken gegen den möglichen Sturz des ägyptischen Machthabers als Folge der Krise. ${ }^{9}$ Die Amerikaner, so Adenauer, hätten den ägyptischen Präsidenten im letzten Moment vor den Israelis gerettet. ${ }^{10}$

Die Sowjetunion und den Kommunismus betrachtete er als große Gefahr und warnte vor russischen Plänen, ihre Ideologie auch im Nahen Osten zu verbreiten. Die Amerikaner seien sich nicht bewußt, daß dies zu einer Umklammerung Euro-

6 NEW YORK TIMES vom 1. 11. 1956; Ausarbeitung von Kurt R. Grossmann „Das Luxemburg-Abkommen und der Nahostkonflikt" vom 1.11. 1956, CZA, Z6/1020; Ebo Rothschild an von Brentano vom 2.11. 1956, BArch, N 1239/183; Krekeler an das AA vom 2. 11. 1956; Aufzeichnung von van Scherpenberg an den Bundesminister über den Staatssekretär vom 2. 11. 1956, PA, 708, 82.04, 92.19.

7 Herbert Lehman an Herbert Hoover, Jr., Außenminister, vom November 1956, YIVO, AJC, FAD-1, Box 69, Israel; Krekeler an das AA vom 2. 11. 1956, PA, 708, 82.04, 92.19; Blankenhorn an Adenauer vom 3. 11. 1956, BArch, N 1351, Bd. 69. Mindestens ein hoher Vertreter des amerikanischen State Department bestritt die Forderung, wonach die Schilumim einzustellen seien: Shimshon Arad an Abba Eban vom 13. 11. 1956, ISA, 2543/11.

8 5.11. 1956, 17.11. 1956, Stiftung Bundeskanzler-Adenauer-Haus, Rhöndorf, I-010, Nr. 022/1, Nr. 001/1; ADENAUER, Teegespräche 1955-1958, Nr. 11, S. 134-143; Aufzeichnung über das Gespräch von Adenauer mit Botschafter Dr. Conant und Senator Green vom 17. 11. 1956, BArch, N 1351, Bd. 69.

9 Tagebucheintrag vom 12.11. 1958, BGD, BGD.

10 Aufzeichnung Blankenhorns vom 6. 11. 1958, BArch, N 1351, Bd. 69; Fischer an das Außenministerium, den Generaldirektor und den stv. Generaldirektor betr. Bericht über das Treffen mit Adenauer am 16. 6. 1958 vom 18. 7. 1958, ISA, 3309/25. 
pas aus südöstlicher Richtung her führen würde, warnte der Kanzler. Bei der Konfrontation mit dem Kommunismus im östlichen Mittelmeerraum stehe immerhin die Sicherheit Europas auf dem Spiel. Die Politik der USA, so Adenauer, widerspreche vitalen europäischen Interessen, und die Bundesrepublik sei deshalb dazu verpflichtet, ihren europäischen Verbündeten beizustehen. ${ }^{11}$ Israel sei von Ägypten bedroht, das wiederum von der Sowjetunion unterstützt werde. Daraus folgerte der Bundeskanzler, daß Israel indirekt auch von den Russen bedroht sei.12 Adenauer betrachtete Israel als Teil Europas, zumindest was die Sicherheit des Kontinents anbetraf. Die ausgezeichnete israelische Armee wertete er als Teil der europäischen Verteidigung.

Die Haltung des Kanzlers und später auch seines Verteidigungsministers Franz Josef Strauß gegenüber Israel waren von einer Mischung aus Nützlichkeitsdenken und Pragmatismus aber auch von moralischen Erwägungen geprägt. Der jüdische Staat war in ihren Augen ein für die Verteidigung Europas günstig plazierter Militärfaktor. Außer vielleicht von Ben Gurion wurde diese neue Perspektive in Israel vorerst von niemandem zur Kenntnis genommen. Adenauer und von Brentano betonten ihre Sympathie für das belagerte Israel. In einem Gespräch mit dem israelischen Botschafter Maurice Fischer in Paris etwa zwei Jahre später drückte der Bundeskanzler die Hoffnung aus, daß Israel in Zukunft über Nuklearwaffen verfügen werde. ${ }^{13}$ Die Bundesrepublik nahm nach außen eine neutrale Haltung ein, indem sie sich ostentativ von Ägypten und den Feinden Ägyptens, aber auch von der amerikanischen Linie distanzierte.

Als die Debatte über die mögliche Einstellung der Schilumim auch Jerusalem erreichte, wandten sich Ben Gurion und Finanzminister Eschkol in schriftlichen Appellen an ihre deutschen Amtskollegen. ${ }^{14}$ Dies bot Adenauer die Gelegenheit, seine Sympathie für Israel auszudrücken, zumindest was die Verwicklung des jüdischen Staates in die Suezkrise anbetraf. Shinnar flog umgehend nach Bonn und wurde vom Kanzler sofort empfangen. Dieser versicherte ihm, daß der Güterstrom ungehindert weiter fließen werde, solange nicht Militärgüter involviert seien. Außerdem bekräftigte Adenauer seine Unterstützung für die Militäraktion und gab der Hoffnung Ausdruck, sie möge Israels Probleme lösen..$^{15}$ Am folgenden Tag wurde die Erklärung hinsichtlich der Fortsetzung der Schilumimlieferun-

11 Von Brentano an Adenauer vom 2. 11. 1956, BArch, N 1239, Bd. 156; Aufzeichnung Blankenhorns vom 6. 11. 1956, BArch, N 1351, Bd. 69; Aufzeichnung über das Gespräch von Adenauer mit Botschafter Dr. Conant und Senator Green vom 17.11. 1956, BArch, N 1351, Bd. 69.

12 Aufzeichnung über das Gespräch von Adenauer mit Botschafter Dr. Conant und Senator Green vom 17. 11. 1956, BArch, N 1351, Bd. 69; Aktennotiz für die Vorstandsitzung vom 5. 11. 1956, ACDP, VIII-001-1502/1-2.

13 Fischer an das Außenministerium, den Generaldirektor und den stv. Generaldirektor betr. Bericht über das Treffen mit Adenauer am 16.6. 1958 vom 18. 7. 1958, ISA, 3309/25.

14 Ben Gurion an Adenauer vom 1. 11. 1956, Eschkol an Schäffer vom 1. 11. 1956, ISA, 2543/ 11. Soweit dem Autor bekannt, handelte es sich hierbei um das erste Schreiben in der längeren Korrespondenz zwischen Adenauer und Ben Gurion.

15 Shinnar an Ben Gurion vom 4. 11. 1956, ISA, 2543/11; Telegramm Shinnars an die Minister des Äußeren, der Finanzen, Handel und Industrie und den Direktor der Schilumimgesellschaft vom 3. 11. 1956, ISA, II 183-12-chet. 
gen veröffentlicht. ${ }^{16}$ Diese Nachricht beseitigte die Ungewißheit und sorgte bei deutschen Banken, deutschen Industriebetrieben und Produzenten von Schilumimgütern für Erleichterung. ${ }^{17}$ Die Korrespondenz der beiden Regierungschefs ging weiter. Sie war sowohl äußerlich als auch inhaltlich äußerst formal gehalten und enthielt nur selten eine persönliche Note. Gelegentlich übermittelten persönliche Boten Grüße, die auch fremde Ohren erreichten.

Während des Suezfeldzugs besetzten israelische Truppen den Gazastreifen, die Basis der Fedajin, und Scharm-el-Scheich, die den Golf von Akaba dominierende Südspitze der Halbinsel Sinai. Die UNO und die USA forderten ultimativ den Rückzug der Israelis. Sie drohten dem jüdischen Staat erneut mit einem Embargo und versuchten, Bonn zur Einstellung der Schilumimlieferungen zu bewegen. Wie bereits beim Ausbruch der Suezkrise weigerte sich die Bundesregierung jedoch auch diesmal, „eine moralische Frage mit diplomatischen Zielen zu vermengen“. 18 Daran konnte auch ein angebliches Schreiben Eisenhowers an Adenauer nichts ändern, in dem der amerikanische Präsident den Bundeskanzler persönlich aufgefordert haben soll, Ben Gurion zur Ordnung zu rufen. Adenauer war nicht bereit, die Schilumim als Druckmittel einzusetzen. ${ }^{19}$ Die Gerüchte über ein solches Schreiben wurden später dementiert, hielten sich aber trotzdem hartnäckig aufrecht. Die Bundesrepublik war starkem moralischem Druck ausgesetzt, als Nichtmitglied der UNO allerdings nicht an die Beschlüsse dieser Organisation gebunden. Wie zuvor brach die Bundesrepublik auch diesmal die einheitliche antiisraelische Front bzw. das Embargo gegen Israel, das ohne deutsche Mitwirkung nicht effektiv sein konnte. Die israelische Regierung wußte die Haltung des Kanzlers zu würdigen. ${ }^{20}$ Sicherheitshalber bat der westdeutsche Botschafter in Washington Goldmann, der israelischen Regierung den Wunsch der Bundesregierung zu übermitteln, Israel möge im Streit um die von Israel besetzten Gebiete eine gemäßigte Haltung einnehmen, und drückte die Hoffnung aus, daß es zu einer befriedigenden Lösung kommen werde. ${ }^{21}$

Der Politik der Bundesregierung und vor allem der Entschlossenheit des Kanzlers war ein langfristiger Effekt beschieden. Mit ihrer Reaktion auf die sowjetischen Drohungen in der Suezkrise trug die Bundesrepublik wesentlich zur Verwandlung des israelisch-arabischen Konflikts zu einem weiteren Schauplatz der Ost-West-Konfrontation bei, diesmal nicht zu ihrem Nachteil. Obwohl der Verlauf der Suezkrise deutlich gemacht hatte, daß Alleingänge der europäischen Staaten infolge ihre Schutzbedürftigkeit durch den amerikanischen Nuklearschirm nicht erfolgreich sein konnten, zeigte sich nach dem Ende der Suezkrise bei den folgenden NATO-Verhandlungen ein Perzeptionswandel in der amerikanischen

16 Ma'ARIv (Tel Aviv) vom 4. 11. 1956; SüdDEUTSCHe ZeITUNG (München) vom 6. 11. 1956.

17 Aufzeichnung van Scherpenbergs an den Bundesminister über den Staatssekretär vom 2. 11. 1956, PA, 708, 84.04, 92.19 .

18 Außenministerium, Büro für Europäische Angelegenheiten, an den Außenminister vom 26. 2. 1957, USNA, 662A/2-2657.

19 Ilsar an Eytan vom 3. 3. 1957, ISA, 3090/25.

20 Ilsar an den Generaldirektor vom 3. 3. 1957, ISA, 3099/26; Bericht der US-Botschaft in Bonn vom 14. 3. 1957, USNA, 662A.84A/3-1457.

21 Ilsar an Shinnar vom 17. 3. 1957, ISA, 3099/26. 
Außenpolitik. ${ }^{22}$ Die israelischen Schritte wurden immer mehr aus der Perspektive des Kalten Krieges und immer weniger als Teil eines begrenzten Regionalkonflikts betrachtet. Israel glaubte, daß die riesigen Waffenlager, die Ägypten in der Sinaiwüste angelegt hatte, gar nicht für den Eigenbedarf bestimmt sein könnten. Vielmehr würde dieses Kriegsgerät im Bedarfsfall der sowjetischen Armee zur Verfügung stehen, wandten die Israelis bei ihren Bemühungen um eine Verständigung mit der NATO ein. Bonn übernahm dieses Argument und betrachtete Israel als wichtigen Sicherheitsfaktor für den Westen. Dies verlieh den deutsch-israelischen Beziehungen eine neue Dimension, die über den Holocaust-Komplex hinausging.

Im Rückblick betrachtet, leitete die Suezkrise auf weltpolitischer wie auf regionaler Ebene, im militärischen und militärstrategischen Bereich, ja sogar in der Geisteshaltung eine neue Ära ein. Europäischen Klischees gemäß kampfunfähig und drückebergerisch, galten die Juden nun plötzlich als hervorragende Soldaten. Westdeutsche Zeitungen und Militärkreise bezeichneten den Sinaifeldzug als „Blitzkrieg“. Die Operation beeindruckte besonders auch rechte, konservative und militärische Kreise in der Bundesrepublik und leitete die Zusammenarbeit zwischen der israelischen Armee (Israel Defense Forces - IDF) und der Bundeswehr ein. Ein weiteres Ergebnis des Sinaifeldzugs war die zunehmende Entfremdung liberaler und linker Kreise gegenüber Israel, bis zum offenen Bruch Ende der sechziger Jahre. ${ }^{23}$ Nachdem sich der Sturm um den Sinaifeldzug gelegt hatte, kehrten die Israelis zur unmittelbaren bilateralen Tagesordnung zurück, nämlich zur Frage der diplomatischen Beziehungen. Jerusalem setzte auf direkte Kontakte.

\section{Diplomatische Beziehungen versus Finanzhilfe und Waffengeschäfte}

Der tiefere Grund des westdeutschen Widerwillens gegen die Aufnahme diplomatischer Beziehungen wurde in Israel nicht wahrgenommen. Man betrachtete die Schwierigkeiten als vorübergehend und führte sie auf antiisraelische Tendenzen in den mittleren und unteren Rängen des Auswärtigen Amts zurück. In Jerusalem sah man hierin ein Problem, das sich mit Überzeugungsarbeit an den entscheidenden Stellen würde beheben lassen. Ansprechpartner sei der Kanzler, betonte Shinnar unentwegt. Da Adenauer die Aufnahme von Beziehungen zu Israel in der Öffentlichkeit uneingeschränkt unterstützte, wurde versucht, das Problem möglichst noch in seiner Amtszeit zu lösen. Trotz entschlossener sozialdemokratischer Unterstützung der Aufnahme von Beziehungen zu Israel waren einige Diplomaten, darunter auch der den Sozialisten eher abgeneigte Shinnar, überzeugt, daß sich die Chancen für die Aufnahme solcher Beziehungen bei einem etwaigen

22 Aufzeichnung vom 14. 11. 1956, BArch, N 1351/69.

23 William L. Hamilton, US-Botschaft in Tel Aviv, an den Außeminister vom 14.3. 1957, USNA, 662A.84/3-1457. 
Rücktritt von Adenauers Kabinett verringern würden. ${ }^{24}$ Ein Glaube, den der gewiefte Kanzler bewußt zu nähren verstand. Adenauer unterstützte von Brentanos Haltung in der Deutschen Frage, zur Hallsteindoktrin und zur Wiedervereinigung, die, so scheint es, dem Bundesaußenminister mehr am Herzen lag als ihm selbst. ${ }^{25}$ Insofern dürfte er auch den Standpunkt des Auswärtigen Amts geteilt haben, wonach Beziehungen zu Israel die Chancen der Wiedervereinigung beeinträchtigen würden. So zwang er seine Diplomaten nicht, die Annäherung an Israel zu beschleunigen. Die Israelis durchschauten des Kanzlers Manöver nicht. Selbst später, nachdem bereits Zweifel aufgekommen waren, vermieden die Israelis vorschnelle Schlußfolgerungen und direkte Angriffe auf Adenauer. Dies hielt israelische Regierungsvertreter allerdings nicht davon ab, von Brentano und andere deutsche Politiker und Diplomaten in internen Dokumenten zu kritisieren und zu verunglimpfen. Leo Kohn, ein altgedienter politischer Beirat im Außenministerium, ritt Mitte 1958 eine scharfe Attacke gegen von Brentano und beschuldigte ihn, mit jenen Industrie- und Handelskreisen zusammenzuarbeiten, die seinerzeit Hitler unterstützt hätten. ${ }^{26}$ Es gab keine schlimmere Anschuldigung im politischen Vokabular in Israel als die Kollaboration mit Hitler. Solche verbalen Feldzüge zeugten von wachsender Ungeduld auf israelischer Seite.

In den folgenden Jahren war es die israelische Regierung, die auf diplomatische Beziehungen drängte, und das Auswärtige Amt, das seinen ablehnenden Standpunkt aufrecht erhielt. Ein Beamter des Auswärtigen Amts schilderte diesen Vorgang einem Vertreter der britischen Botschaft wie folgt: „Shinnar trifft den Außenminister alle sechs Monate und verlangt jedes Mal diplomatische Beziehungen. Dabei wird ihm jeweils erklärt, daß der momentane Zeitpunkt dafür nicht geeignet sei. ${ }^{27}$ Das halbjährliche Treffen wurde zum hohlen Ritual, zur Posse mit zwei Darstellern, die beide ihre Rolle perfekt beherrschten. Die deutsche Seite bewies mit ihren Ausreden viel Phantasie. Es ging längst nicht mehr nur um die arabische Anerkennung der DDR, die Verweigerung der Beziehungen zu Israel wurde quasi zum Selbstzweck.

So teilte von Brentano Shinnar mit, daß die Anerkennung der DDR durch Syrien kein ausreichender Grund für die Aufnahme diplomatischer Beziehungen zu Israel sei, da andere arabische Staaten Damaskus nicht zwingend folgen würden. ${ }^{28}$ Am 16. Mai 1957 erklärte der Bundesaußenminister gegenüber Goldmann, die Aufnahme diplomatischer Beziehungen sei möglich, sobald an Israels Grenzen mindestens zwei Monate (Waffen-)Ruhe herrsche. ${ }^{29}$ Auch beim Auftakt von Handelsgesprächen zwischen der Bundesrepublik und der Sowjetunion im Mai 1957 wurde auf deutscher Seite die Aufnahme von Beziehungen mit Israel in Aus-

24 Bentzur, Wien, an den Generaldirektor vom 3. 1. 1957, ISA, 3099/26.

25 KOSTHORST, Brentano und die deutsche Einheit, S. 88-93; FOSCHEPOTH, Westintegration, S. 29-60.

26 Leo Kohn an Jacob Herzog, israelische Botschaft in Washington, D.C. vom 15. 6. 1958, ISA, 3309/25.

27 Die britische Botschaft in Bonn an die Abteilung Levante vom 6. 10. 1958, PRO, FO 371/ 134275, VR 10318/6.

28 Ilsar an den Abteilungsleiter für Westeuropa vom 5. 11. 1957, ISA, 3309/25.

29 Goldmann an Meir vom 16. 5. 1957, ISA, 3309/25. 
sicht gestellt, dann aber mit dem Scheitern dieser Gespräche wieder bis auf weiteres verschoben. ${ }^{30}$ Der Abbruch der diplomatischen Beziehungen zwischen Bonn und Belgrad als Folge der Anerkennung der DDR durch Jugoslawien wurde von der Bundesregierung dann als weiterer Vorwand für den Aufschub der Aufnahme von Beziehungen zu Israel genutzt: Die westdeutsche Politik der Annäherung gegenüber dem Osten hätte, so die Argumentation des Auswärtigen Amts gemäß Shinnar, auch einen ähnlichen Schritt im Nahen Osten gerechtfertigt, doch Marschall Tito habe sie zunichte gemacht. ${ }^{31}$ Der gute Ruf des Staates Israel liege dem Auswärtigen Amt sehr am Herzen. Eine Anerkennung der DDR durch die Araber als Folge des Austauschs von Botschaftern zwischen Bonn und Jerusalem, so Shinnar, würde den Zorn der deutschen Öffentlichkeit auf Israel lenken, was das Auswärtige Amt unter allen Umständen verhindern wolle. ${ }^{32}$ Eine weitere Botschafterkonferenz in Berlin im Mai 1957 bekräftigte die Beschlüsse der Istanbuler Konferenz. ${ }^{33}$

Bei den ablehnenden Erklärungen, die Shinnar von deutscher Seite gegeben wurden, handelte es sich offensichtlich um bloße Vorwände. Der israelische Diplomat war, ohne es wahrzunehmen, zum unfreiwilligen Hauptdarsteller einer endlosen Farce geworden. Jahr für Jahr wiederholte sich ein und dasselbe Ritual: In der Presse erschienen Berichte über die angeblich bevorstehende Aufnahme diplomatischer Beziehungen zwischen der Bundesrepublik Deutschland und dem Staat Israel, worauf die Außenministerien der arabischen Staaten und die arabischen Botschaften in der Bundesrepublik das Auswärtige Amt und die westdeutschen Botschaften mit Protesten überschwemmten. Die westdeutsche Diplomatie reagierte wiederum mit einer Flut von Dementis, und die arabischen Staaten antworteten ihrerseits mit einer Welle von Verlautbarungen, in denen sie ihre Erleichterung über die „guten Nachrichten“ ausdrückten. Darauf kehrte im Auswärtigen Amt wieder Ruhe ein, bis zu Shinnars nächstem Vorstoß.

Gleichzeitig spielte sich in Israel ein ähnliches Ritual ab, wenn auch in umgekehrtem Sinne: Zeitungsberichte über die bevorstehende Aufnahme diplomatischer Beziehungen zur Bundesrepublik riefen die parlamentarische Opposition auf den Plan und provozierten größere Debatten im Plenum. So veranlaßte die rechtsgerichtete Cherut-Partei am 15. Juli 1957 eine größere Debatte in der Knesset, nachdem Ben Gurion auf einer Pressekonferenz am 28. Juni zum ersten Mal öffentlich seiner Hoffnung Ausdruck gegeben hatte, daß Israel und die Bundesrepublik demnächst diplomatische Beziehungen aufnehmen werden. ${ }^{34}$ Im Verlaufe dieser Knesset-Debatte sprach sich Ben Gurion für die Aufnahme von Beziehungen mit der Bundesrepublik aus und rief zu deutschen Investitionen in Israel sowie zur Zusammenarbeit beider Länder auf weiteren Ebenen auf. Ein Aufruf, den

30 Shinnar an den Generaldirektor vom 16.5. 1957, ISA, 3309/25.

31 Shinnar an den Generaldirektor vom 1. 11. 1957; Shinnar an Fischer vom 5. 5. 1957, ISA, $3309 / 25$.

32 Shinnar an den Generaldirektor vom 1. 11. 1957, ISA, 3309/25.

33 Arad an den Außenminister vom 15. 5. 1957; Shamai Kahana, persönlicher Assistent von Abba Eban, an Nahum Goldmann vom 20. 5. 1957, ISA, 3309/26.

34 Baxter, US-Botschaft in Tel Aviv, an den Außenminister vom 2.7. 1957, USNA, 662A.84A/7-257. 
der israelische Ministerpräsident im selben Jahr und auch später noch mehrmals wiederholte, nicht ohne jeweils im positiven Sinne zu betonen, daß die Bundesrepublik ein „Neues Deutschland“ verkörpere. ${ }^{35}$

Enthüllungen der israelischen Presse über Verhandlungen zur Aufnahme diplomatischer Beziehungen kamen dem Auswärtigen Amt höchst ungelegen. Die Abteilung 3 zeigte sich besorgt über die arabischen Proteste, besonders über die scharfen syrischen Attacken. Die Israel-Mission in Köln wurde aufgefordert zu intervenieren, und die israelische Regierung versprach prompt, sich um die Entfernung des Themas aus den Schlagzeilen zu bemühen. Bemerkenswert ist ohne Zweifel der Umstand, daß die Israelis bereit waren, die fast antiisraelischen Forderungen des Auswärtigen Amts umzusetzen. Der israelische Historiker David Shaham behauptet, oberste politische Stellen in Israel hätten anläßlich einer geheimen Absprache im Sommer 1957 beschlossen, das Thema diplomatische Beziehungen zur Bundesrepublik vorläufig von der Tagesordnung zu nehmen. ${ }^{36}$ Dies schien das israelische Außenministerium und auch Shinnar allerdings nicht davon abzuhalten, sich weiter offen mit dieser Frage zu beschäftigten. Ganz im Gegensatz zu Ben Gurion, der ihre Erwähnung in seinem schriftlichen und mündlichen Verkehr mit Bundeskanzler Adenauer vermied. ${ }^{37}$ Für den Pragmatiker Ben Gurion hatten die diplomatischen Beziehungen ohnehin nicht oberste Priorität. Er war vielmehr an praktischer deutscher Hilfe im Finanz-, Wirtschafts- und Rüstungsbereich interessiert. Der Kanzler nahm offenbar einen ähnlichen Standpunkt ein, so daß die beiden Regierungschefs in dieser Frage stillschweigend übereinstimmten. Goldmann verfolgte einen offeneren und direkteren Kurs. Mit viel persönlichem Charme und auf geistreiche Art gelang es ihm, den Kanzler in jenen Fragen zu überzeugen, die er als besonders wichtig erachtete. Wenn es Goldmann lohnend erschien, erwähnte er auch das Thema diplomatische Beziehungen, ohne jedoch den Kanzler zu einer verbindlichen Antwort zu drängen.

Ben Gurion setzte beim Meinungsaustausch mit Adenauer besonders bei wichtigen Fragen auch auf persönliche Gesandte. 1957 übernahm diese Rolle Josephthal, 1958 Maurice Fischer und 1962 - worauf später noch näher eingegangen wird - Shimon Peres, damals stellvertretender Verteidigungsminister. ${ }^{38}$ Josephthal hatte den Auftrag, um Adenauers Zustimmung für den damals erwogenen israelischen Beitritt zur NATO zu werben oder zumindest um gewisse westliche Garantien für die Grenzen des Staates Israel bzw. um Sicherheitsgarantien für den jüdischen Staat. Das Treffen zwischen Josephthal und dem Kanzler am 12. Dezember 1957, an dem auch Shinnar teilnahm, stiftete große Verwirrung und gab Anlaß

35 ISRAEL INFORMATIONSDIENST (Köln), Nr. 52 vom August 1957; Die Welt (Hamburg) vom 17.7. 1957; Zitate aus Berichten der israelischen Tageszeitungen DAWAR und HAARETZ, in denen Ben Gurion für seinen Weitblick gelobt wird: die britische Botschaft in Tel Aviv an die Abteilung Levante vom 18. 7. 1957, PRO, FO 371/128094. Der britische Diplomat berichtete über die positive Reaktion der Presse und der Öffentlichkeit in Israel und äußerte die Meinung, daß die Aufnahme diplomatischer Beziehungen kurz bevorstehe.

36 SHAHAM, Israel, S. 173.

37 Ben Gurion an Adenauer vom 18. 9. 1957, PA, 708, 52.04, 92.19.

38 Shinnar an Eytan vom 19. 11. 1956, ISA, 3309/25. 
zu zahlreichen Gerüchten. Josephthal übergab Adenauer offenbar ein Memorandum, das auf Israels Probleme und Forderungen aufmerksam machte. ${ }^{39}$ Fischers Gespräch mit Adenauer am 17. Juli 1958 im Beisein von Shinnar und Globke40 fand inmitten von Spannungen im Nahen Osten und Unruhen im Irak, in Jordanien und im Libanon und während der westlichen Militärintervention in der Region statt. ${ }^{41}$ Fischer erläuterte dem Kanzler den israelischen Standpunkt zur westlichen Politik im Nahen Osten und zur möglichen Rolle der Bundesrepublik in der Region. Adenauer bekräftigte seinerseits den guten Willen der Bundesregierung und ihre Bereitschaft, Israel zu unterstützen.

Die beiden Missionen waren indirekte Resultate des Suezfeldzugs, nach dem Israel sich dazu entschlossen hatte, die Beziehungen mit Bonn zu vertiefen. Ben Gurion suchte den Kontakt mit Adenauer auf politischen und nicht auf diplomatischen Wegen. Er wollte persönlich auf den Kanzler einwirken und dabei seine Beziehungen zum westdeutschen Regierungschef intensivieren. Möglicherweise erwartete Ben Gurion dabei zu viel. Im Zwiespalt zwischen Wiedervereinigungsbestrebungen und der amerikanischen Entspannungspolitik sowie zwischen amerikanischen und französischen Strategiekonzepten hin und her gerissen, waren Adenauer die Hände praktisch gebunden. Der Wettstreit zwischen den verschiedenen weltpolitischen Strategien des Westens betraf auch den Nahen Osten und hatte insofern auch Einfluß auf die arabische Politik gegenüber den beiden deutschen Staaten. Dem Auswärtigen Amt war es zudem offenbar gelungen, den Bundeskanzler in israelischen Angelegenheiten zu einer passiveren Haltung zu bewregen. Ben Gurions Enttäuschung über Adenauers Passivität kam im Tagebucheintrag vom 30. September 1958 zum Ausdruck: „Auch Hitler hat sich auf die Demütigung des deutschen Volkes berufen. Doch muß denn Deutschland immer so bleiben wie [zu] Hitler[s Zeiten]?"

Westdeutsche Quellen behaupteten wiederholt, die USA hätten der Bundesrepublik von der Aufnahme von Beziehungen zu Israel abgeraten, um ihren Sonderstatus im Nahen Osten nicht zu gefährden. Im Vorfeld eines Treffens zwischen Bundesaußenminister von Brentano und seinem amerikanischen Amtskollegen Dulles im März 1957 bat die israelische Regierung den amerikanischen Außenminister über das State Department, sich bei von Brentano für die Aufnahme diplomatischer Beziehungen zwischen der Bundesrepublik und Israel einzusetzen. Dulles übermittelte die israelische Bitte seinem Gegenüber ohne Kommentar, und dieser nahm sie ebenso kommentarlos zur Kenntnis. ${ }^{42}$ Davon abweichend be-

39 Shinnar an Fischer (einschließlich Kopie des Memorandums) vom 30. 4. 1958, ISA, 3309/ 25. Zu Josephthals Mission: Notizen vom 23. 12. 1957, PRO, FO 371/128094; die britische Botschaft in the Tel Aviv an die Abteilung Levante vom 23. 12. 1957, PRO, FO 371/ 134296; Shinnar an den Generaldirektor vom 29.12. 1957, ISA, 3309/25; Tagebucheinträge vom 18., 19. und 29. 12. 1957, BGA, BGD; HATZOFEH (Tel Aviv) vom 18. 12. 1957; FRANKFURTER ALLGEMEINE ZEITUNG vom 19.12.1957.

40 Fischer an Meir, an den Generaldirektor und seinen Stellvertreter vom 18. 7. 1958, ISA, 3309/25; zu den Vorbereitungen auf dieses Gespräch: Tagebucheintrag Ben Gurions vom 17. 6. 1958, BGA, BGD; Fischer an Harman vom 17. 6. 1958, ISA, 3309/25.

41 BaILEY, Jordan's Palestinian Challenge, S. 56-62; SHWADRAN, Jordan, S. 364-381.

42 Shinnar an Eytan vom 2. 3. 1957, ISA, 3099/25; Notiz von Zeev Argaman, israelische Bot- 
hauptete die israelische Tageszeitung HaAretz am 13. Juni 1957, die USA hätten der Bundesrepublik geraten, sich mit der Aufnahme diplomatischer Beziehungen zu Israel Zeit zu lassen. Man darf annehmen, daß dieser Ratschlag auf den Einfluß antiisraelischer Elemente im State Department zurückging. Insgesamt dürfte das amerikanische Außenministerium seine Beschlüsse aber eher individuell von Fall zu Fall und gemäß der Politik des jeweiligen Präsidenten gefällt haben.

Israel sah die Beziehungen zur Bundesrepublik offenbar in einem viel größeren Zusammenhang. In einem Gespräch mit Walter Eytan am 5. Februar 1957 schilderte Ben Gurion seine Vorstellung der israelisch-deutschen Beziehungen wie folgt: „Wir sollten mit Deutschland Beziehungen wie mit Frankreich unterhalten. “43 "Das ist ein klares Wort", kommentierte Eytan. Zu jenem Zeitpunkt, das heißt kurz nach dem Suezfeldzug, standen die Beziehungen zwischen Frankreich und Israel immer noch in voller Blüte. Frankreich unterstützte Israel in der Atomforschung, im geheimdienstlichen Bereich sowie in der Militärforschung und lieferte dem jüdischen Staat moderne Waffensysteme. Zudem unterhielten die beiden Staaten enge Beziehungen in den Bereichen Handel, Finanzen und Kultur. Erst nach der Wahl von Charles de Gaulle zum Präsidenten der fünften Republik und dem Ende des algerischen Unabhängigkeitskrieges, als sich eine Annäherung zwischen Frankreich und der arabischen Welt abzeichnete, begann sich das Verhältnis zwischen Israel und Frankreich allmählich zu verschlechtern. Die Bundesrepublik sollte nun, nach Ben Gurions Vorstellungen, Frankreichs Rolle als bester Freund Israels übernehmen.

Die israelische Regierung bemühte sich um französische und deutsche Investitionen für den Bau einer Pipeline und einer Eisenbahnlinie zwischen dem Roten Meer und der Mittelmeerküste zur Umgehung des Suezkanals. Die Israelis versuchten die Europäer davon zu überzeugen, daß dieses Doppelprojekt sie von der Abhängigkeit von Nasser befreien würde. ${ }^{44}$ Dazu beantragte die israelische Regierung in Bonn einen Kredit, der mit den beiden letzten Schilumimraten verrechnet werden sollte. Ben Gurion maß der Bundesrepublik bei der Festigung der Beziehungen mit der NATO eine Schlüsselrolle bei. Israel und die Bundesrepublik trieben regen gegenseitigen $\mathrm{Handel}$ mit Rüstungsgütern, und auch der beiderseitige Handel mit nichtmilitärischen Gütern außerhalb des Schilumimrahmens war im Steigen begriffen. Zudem erstreckte sich die Zusammenarbeit zwischen der Bundesrepublik und Israel bis 1957 auf manch weitere Bereiche wie Justiz, Entschädigung, Kultur und Hochschulen. Es gab erste Ansätze von Tourismus, und später kamen weitere Bereiche hinzu. Obwohl sich die Beziehungen zwischen der Bundesrepublik und Israel äußerlich stark von den französisch-israelischen Beziehungen abhoben, hatten sie sich bereits sehr stark entwickelt, und zwar genau in die Richtung, die Ben Gurion vorgezeichnet hatte.

schaft, für Berry Lampton, NEA, und Donald G. Bergus, NE, vom 6. 3. 1957, USNA, 662A.84A/3-657.

43 Eytan an Shinnar vom 7. 2. 1957, ISA, 3099/25.

44 Shinnar an Eschkol und Saphir vom 10. 12. 1956, ISA, 3100/4; Shinnar an Eban vom 7. 12. 1956; PA, 708, 82.04, 92.19; Goldmann an Blankenhorn vom 9. 1. 1957, CZA, Z6/2001. 
1957 kam es zu einer Vertiefung der militärischen Zusammenarbeit zwischen beiden Ländern. Nachdem die westdeutsche Industrie und der westdeutsche Markt den israelischen Verteidigungsbedürfnissen bereits Jahre gute Dienste geleistet hatten, gelang es, diese Beziehungen in jenem Jahr unter dem Eindruck des Suezfeldzugs endlich zu institutionalisieren. Strategisch-politische Erwägungen und die intensive Beschäftigung der Israelis mit militärischen Fragen hatten zu einer allmählichen Annäherung zwischen den höchsten politischen Stellen der Bundesrepublik und Israels beigetragen. Daran war auf israelischer Seite der intelligente, ambitionierte und ränkevolle Ben Gurion-Günstling Shimon Peres beteiligt, der zu jener Zeit als Generaldirektor des Verteidigungsministeriums amtierte. Peres war von einer Schar von Ben Gurion-Beratern umgeben, die sich eher für praktische Aufgaben als für Ideologie interessierten und deshalb den Beinamen „Bitsuistim“ (Vollstrecker) erhielten. Der militärische Kopf dieser von Ben Gurions Zukunftsvisionen geprägten Gruppe war General Moshe Dayan. Auf deutscher Seite stand Peres der neue Bundesverteidigungsminister Franz Josef Strauß gegenüber. Der intelligente, impulsive, analytisch denkende und entscheidungsfreudige Strauß fand mit Peres schnell eine gemeinsame Sprache. Strauß hielt seinen Chef über die Kontakte zu Peres auf dem laufenden, um sich abzusichern. Dennoch scheint der Bundeskanzler Bedenken gehabt zu haben, denn er zog es vor, nicht im einzelnen informiert zu werden. ${ }^{45}$

Die Verhandlungen fanden also direkt zwischen dem israelischen Verteidigungsministerium und dem bundesdeutschen statt. Nur die politischen Entscheidungsträger und höhere Beamte hatten davon Kenntnis. Der hochrangige israelische Diplomat Maurice Fischer erfuhr dazu nähere Einzelheiten erst während seines Aufenthalts in Köln im Sommer 1958. Zum engeren Kreis der Eingeweihten auf deutscher Seite gehörten Hallstein und der Direktor der politischen Abteilung des Auswärtigen Amtes, Wilhelm Grewe. Die Herren der Abteilung 3 wurden dagegen völlig im dunkeln gelassen, und als die Angelegenheit publik wurde, war es für Gegenmaßnahmen bereits zu spät. ${ }^{46}$

Die militärische Zusammenarbeit und der gegenseitige Waffenhandel wurden geheim gehalten. Einzelheiten, die dennoch durchsickerten, entgingen der Aufmerksamkeit der Öffentlichkeit, die sich über die größeren Zusammenhänge damals noch kein Bild machen konnte. Rüstungslieferungen, Finanzhilfe und diplomatische Beziehungen standen in einem engen Zusammenhang, wobei der jeweilige Stellenwert im Zeitablauf unterschiedlich zu gewichten war. Waffengeschäfte und/oder Finanzhilfe verringerten die Dringlichkeit der Aufnahme diplomatischer Beziehungen. Nach geplatzten Rüstungsgeschäften oder gescheiterten Verhandlungen über Finanzhilfe, rückte die Frage der Aufnahme von diplomatischen Beziehungen jeweils wieder in den Vordergrund.

Der Staat Israel war seit seiner Gründung einem Rüstungsembargo unterworfen und daher gezwungen, sich Waffen auf Umwegen zu beschaffen. Die Vereinigten Staaten lieferten aus politischen Gründen nicht, die Sowjetunion war Israel feindlich gesinnt, und europäische Rüstungsgüter waren teuer. Daher kam der

45 Maurice Fischer an den Generaldirektor vom 2. 7. 1957, ISA, 3099/24.

46 Voigt an Meroz vom 24. 6. 1959, PA, 708, 81.15/0, 92.19. 
Bundesrepublik als möglichem Waffenlieferanten Israels großes Gewicht zu, besonders auch deshalb, weil von ihr besondere Rücksicht für Israels Lage erwartet wurde. Die Bundesrepublik enttäuschte diese Erwartungen nicht. Adenauer und Strauß betrachteten Israel als wichtigen Verbündeten. Sie mißtrauten den verschiedenen sowjetisch bzw. kommunistisch beeinflußten arabischen Staaten. Die übrigen arabischen Staaten waren militärisch unbedeutend oder als Erdölproduzenten auf ausländischen Schutz angewiesen. Mit der massiven Aufrüstung Israels mit modernen Waffen hoffte man, dem sowjetischen Waffenarsenal in Ägypten Paroli zu bieten. Die Kombination von israelischer Kampfbereitschaft und westlichen Waffen wurde als geeignetes Mittel gegen die Gefahr aus dem Osten und Südosten betrachtet. Obwohl die NATO nicht bereit war, Israel in ihre Reihen aufzunehmen, kann man davon ausgehen, daß die Bundesrepublik im Einvernehmen mit dem westlichen Verteidigungsbündnis handelte. Der israelischen Regierung waren die westdeutschen und westlichen Überlegungen bekannt. Israelische Vertreter äußerten oft ihre Bereitschaft, Waffen von jeder Quelle anzunehmen. Führende Mapai-Vertreter betrachteten die Bundesrepublik als Zuflucht in der Not. Nicht zufällig benutzte Ben Gurion wiederholt den Begriff "Neues Deutschland“, um den grundlegenden Wandel Deutschlands seit Hitler zu betonen. Man hatte es nun mit einer europäischen Macht zu tun, die auf Israel angewiesen war, darüber hinaus mit einem Staat mit außergewöhnlichem Potential, der als Verbündeter seiner Lage wegen hoch interessant war.

Die moralischen und ethischen Aspekte der neuen militärischen Allianz zwischen der Bundesrepublik und Israel verdienen ebenfalls Beachtung: Nur rund acht Jahre nach der Kapitulation der Wehrmacht wurde die Bundeswehr ins Leben gerufen. Angesichts der noch frischen Erinnerungen an den Zweiten Weltkrieg wurde sie sofort zu einer Zielscheibe für die sowjetische Propaganda. Nicht als Propaganda, sondern als legitimer Protest wurde dagegen die Opposition der Juden als Hauptopfer des Nationalsozialismus gegen die Wiederbewaffnung Deutschlands empfunden. Gleichzeitig verkaufte der jüdische Staat aber Waffen an die neugeschaffene deutsche Armee. Die Tragweite dieses Schrittes entging weder den Israelis noch den Deutschen und den Amerikanern.77 Daß Ben Gurion zudem die innenpolitischen Implikationen der Rüstungsgeschäfte und besonders des Waffenverkaufs an Westdeutschland unberücksichtigt gelassen haben könnte, ist angesichts der scharfen Polemik sowohl in und außerhalb der Knesset sowie der Regierungskrisen, die solche Waffengeschäfte in der Vergangenheit ausgelöst hatten, ziemlich unwahrscheinlich. Die neue Kontroverse war nicht die erste.

Von Ben Gurion dazu ermächtigt, führte Peres Rüstungsverhandlungen mit westdeutschen Stellen, vor allem mit Strauß. Auf der Tagesordnung stand die Lie-

$47 \mathrm{Vgl}$. die Debatte in der Knesset: KNESSET-PROTOKOLle [Original hebr.], 3. Knesset, 606. Sitzung am 28. 6.-7. 7. 1959, S. 2371-2416; PIKART, Theodor Heuss, S. 450; Vermerk von Dillon, Department of State Instructions vom 15. 7. 1959, USNA, 784A.13/7-1559; Herter, Außenministerium, an die US-Botschaften in Prag und Warschau vom 16.9. 1959, USNA, 784A.15/9-1659 („Der israelische Standpunkt gegenüber der Bundesrepublik [...] in der Frage der Waffenverkäufe an Deutschland zur Verwendung gegen die Kritik an der amerikanischen Unterstützung für die ,revanchistische' Bundesrepublik Deutschland“). 
ferung verschiedener Rüstungsgüter, die aus politischen und wirtschaftlichen Gründen auf regulärem Weg nicht zu haben waren. Es handelte sich vor allem um Unterseeboote, aber auch um Artillerie, spezielle Radareinrichtungen und Raketen. Der Staat Israel suchte seinerseits Märkte für die Maschinenpistole „Uzi“. Außerdem war bekannt, daß sich Strauß und Peres auch für Nuklearwaffen interessierten. Bereits 1957 hatte Peres beschlossen, mit Strauß eine Verständigung über die Lieferung bestimmter Güter durch die Bundesrepublik anzustreben, die anderswo nur mit größten Schwierigkeiten oder gar nicht erhältlich waren. Französische Militärkreise, die gleichzeitig ein enges Verhältnis zu Israel und zur Bundesrepublik pflegten, hatten Peres ermutigt, in Dreiecksverhandlungen einzutreten. 48

Moshe Dayan sagte seine Teilnahme an einem geplanten Treffen zwischen Peres und Strauß ab, an dem er als Partner von Peres und Militärexperte hätte teilnehmen sollen, als die Begegnung im Kabinett erörtert wurde und anschließend durch eine linke Zeitung (ein Koalitionsblatt) an die Öffentlichkeit gelangte. Die Enthüllung sorgte für große Aufregung in Israel, in der Bundesrepublik und auch in der arabischen Welt. In einer stark beachteten Rede in der Knesset rechtfertigte Ben Gurion seine Politik mit dem Argument, Waffen der "dritten Dimension“ seien nur in Deutschland erhältlich, und Israel müsse sich die Waffen dort beschaffen, wo sie erhältlich seien. ${ }^{49}$ Peres fuhr trotz der Schwierigkeiten zum Treffen mit Strauß und nahm an Stelle von Dayan General Chaim Laskov und zwei weitere israelische Vertreter mit. Die Begegnung fand Ende 1957 in Strauß’ Privathaus in Rott am Inn in Bayern statt. Peres und Strauß diskutierten politische und strategische Fragen sowie israelische Waffenkäufe in der Bundesrepublik. Das Gespräch in Rott am Inn legte den Grundstein für zukünftige bilaterale Abkommen über westdeutsche Waffenlieferungen an Israel und für israelische Militärausfuhren in die Bundesrepublik. ${ }^{50}$

Die breite Öffentlichkeit erfuhr von solchen Geschäften erst durch einen Spiegel-Artikel mit der Überschrift „Granaten von Haifa“ vom 6. Juni 1959. Rüstungsgeschäfte mit fremden Ländern, einschließlich derjenigen mit der Bundesrepublik, kamen regelmäßig im Kabinett zur Sprache. Offensichtlich waren sie den Ministern der linken Parteien Mapam und Ahdut Haavoda, wohl auch aufgrund der geschickten Taktik von Ben Gurion, diesmal entgangen. Auch sie erfuhren davon erst durch den Spiegel. Die darüber ausbrechende Kontroverse führte zum Sturz der Regierung und zu Neuwahlen, bei denen es unter anderem auch um die Rüstungsgeschäfte mit der Bundesrepublik ging. Ben Gurion und seine Partei, die Mapai, verteidigten ihr Verhalten, indem sie darauf hinwiesen, daß Deutschland sich geändert habe. Der Handel mit diesem Land sei deshalb legitim. Zudem sei die israelische Rüstungsindustrie auf große Märkte angewiesen,

48 Tagebucheinträge vom 25. 3. und 16. 10. 1957, BGA, BGD; Shinnar an Tswi Dar vom 4. 6. 1957, ISA, II 183-12-chet; GolAN, Shimon Peres, S. 76-78; Interview mit Asher BenNatan vom August 1989; MA'ARIV (Tel Aviv) vom 6. und 9. 6. 1957.

49 KNESSET-PROTOKOLLe [Original hebr.], 2. Knesset, 380. Sitzung am 24. 12. 1957, S. 539.

50 PERES, Kelaa david, S. 47-68; Interview mit Asher Ben-Natan vom August 1989; STRAUSS, Erinnerungen, S. 378-381. 
und die Bundesrepublik bezahle mit Devisen, die wiederum dem Einkauf dringend benötigter Waffen dienten. Im übrigen seien sich Strauß und andere führende westdeutsche Vertreter bewußt, daß die militärischen Bestellungen nicht nur eine direkte Unterstützung der israelischen Rüstungsindustrie bedeuteten, sondern indirekt auch der israelischen Wirtschaft als Ganzes zugute kämen. ${ }^{51}$

Das Ergebnis der Wahl am 3. November 1959 war überwältigend: Ben Gurion errang den größten Wahlsieg seiner politischen Karriere. Die Regierungspartei Mapai erhielt 39\% der Stimmen und konnte sich damit gegenüber den Vorwahlen um sieben Prozent steigern. Die Argumente von Ben Gurions Widersachern hatten die Öffentlichkeit offensichtlich nicht überzeugt. Ob der Wahlsieg von 1959 auch einen Wandel in der Haltung der israelischen Öffentlichkeit zur Bundesrepublik und zur Zusammenarbeit mit ihr bedeutete, bleibt dahingestellt. Fest steht nur, daß die Rüstungsgeschäfte mit der Bundesrepublik nicht das einzige Wahlkampfthema gewesen waren, aber auch, daß die Opposition die Reaktion der Öffentlichkeit auf solche Geschäfte offensichtlich falsch eingeschätzt hatte. Bei einem Teil der israelischen Bürger weckte der Waffenexport nach Deutschland sogar einen gewissen Stolz. Verbreitet war in Israel überdies die Ansicht, daß die Existenz des jüdischen Staates an sich schon „die beste Antwort auf den Nationalsozialismus und auf Hitler" sei. Die Ausfuhr von Waffen nach Deutschland, die von Juden hergestellt worden waren, erfüllte deshalb in Israel viele Bürger mit einer gewissen Befriedigung. Dennoch wäre es verfehlt zu glauben, daß das erwähnte Wahlresultat vom Nachlassen tief sitzender Abneigung, von $\mathrm{Haß}$, Schmerz und anderer negativer Gefühle zeugte. Der praktisch und pragmatisch orientierte Ben Gurion wußte aber die Fähigkeit seiner für Fragen der nationalen Sicherheit und Verteidigung sensibilisierten Bürger richtig einzuschätzen, zwischen Emotionen und pragmatischen Erwägungen zu unterscheiden.

Die Reaktionen in Israel und in der Bundesrepublik waren unterschiedlich. Ben Gurion erhielt zahlreiche Briefe aus dem Volk mit Sympathiebekundungen und Verurteilungen - auch aus Deutschland. Die Briefe aus Deutschland dokumentierten alle ein gewisses Unbehagen. Einige Schreiber kritisierten Ben Gurion und fragten, wie es möglich sei, daß eine Nation von Opfern der Nation der Verfolger Waffen verkaufen könne. Andere drückten Genugtuung darüber aus, daß Deutschland dem jüdischen Staat helfen könne, und einige fügten Solidaritätsbekundungen hinzu. In der eingetroffenen Post befanden sich auch einige $\mathrm{Haß}-$ briefe. ${ }^{52}$ Die westdeutsche Presse reagierte überwiegend negativ und erinnerte an den Grundsatz der Bundesrepublik, keine Rüstungsgüter in Spannungsgebiete zu liefern. Obwohl Strauß als Einzelgänger bekannt war und der jüdische Staat in der Öffentlichkeit erhebliche Sympathien genoß, war die Angelegenheit dennoch von einem gewissen Befremden begleitet. Dem entsprach auch die offizielle Haltung des Auswärtigen Amts. Die israelischen Zeitungen berichteten in der Regel gemäß der Parteivorgaben der jeweiligen Herausgeber.

51 Tagebucheinträge Ben Gurions vom 9. 1. 1958, 18. 2. 1960 und 23. 2. 1960, BGA, BGD;

Zwi Dar, Direktor von TAAS, an Ben Gurion, BGA, Correspondence File, 4. 3. 58.

52 Diverse Briefe aus dem Jahr 1959, BGA, Correspondence File. 
Die Haltung der SPD reflektierte ein erhebliches Maß an Pazifismus. Sie wollte nicht gegen den jüdischen Staat Stellung beziehen, beurteilte die Zusammenarbeit zwischen der jüdischen Seite und dem rechtsgerichteten Strauß jedoch mit großer Skepsis. Zudem stand die Partei dem Waffenhandel negativ gegenüber. ${ }^{53}$ Die SPD vertrat den Standpunkt, daß die Deutschen, denen in der Vergangenheit oft Militarismus und Kriegstreiberei vorgeworfen wurde, sich nicht an diesem „schmutzigen Geschäft" beteiligen sollten.

Die arabischen Sprecher reagierten ungehalten auf die Nachrichten über den Waffenhandel zwischen der Bundesrepublik und Israel. Allein die Zusammenarbeit Westdeutschlands mit dem „Feind“ wurde von ihnen als antiarabische Aggression empfunden. Ob denn die Bundesrepublik ähnliche Waffen nicht anderswo hätte einkaufen können, wollten sie wissen. Die Einnahmen aus diesen Waffenverkäufen würden den Staat Israel im Krieg gegen die Araber unterstützen. Die sowjetische und osteuropäische Presse sowie die jüdischen Institutionen in diesen Ländern stimmten ebenfalls in den Chor der Verurteilungen ein. ${ }^{54} \mathrm{Um}$ die Ägypter zu besänftigen, verpflichtete sich die Bundesregierung auch zum Kauf von Rüstungsmaterial aus ägyptischer Produktion. Auf einer Zusammenkunft westdeutscher Diplomaten in Beirut im August 1959 wurde die Empfehlung gegeben, auf den Waffenhandel in dieser Region zu verzichten. Doch wie in ähnlichen Fällen zuvor wurden die Ratschläge der Diplomaten von den Militärbehörden auch diesmal kaum beachtet. ${ }^{55}$

Ein bemerkenswerter Aspekt des damaligen deutsch-israelischen Verhältnisses war das diplomatische Protokoll. Ab 1954 wurde Shinnar regelmäßig zu offiziellen Anlässen in die Residenz des Bundespräsidenten eingeladen. Der erste solche Auftritt Shinnars löste in Israel großen Unmut aus. ${ }^{56}$ Der israelische Diplomat wurde gerügt, doch seine späteren diplomatischen Auftritte verliefen ohne Reaktionen. Zwischen Bundespräsident Heuss, der besonderes Interesse für den Staat Israel zeigte, und seinem schwäbischen Landsmann Shinnar entstand ein enges freundschaftliches Verhältnis. Im Juli 1953 erhielt der Israeli den persönlichen Rang des Gesandten und im Januar 1958 den persönlichen Rang des Botschafters. Der hohe diplomatische Rang verschaffte Shinnar einen Spitzenplatz auf Empfängen, trotz des niederen Ranges der Israel-Mission, die zusammen mit der finnischen Handelsvertretung den letzten Platz auf der Liste des diplomatischen Protokolls einnahm. Arabische Diplomaten forderten, Shinnar dem Status der IsraelMission gemäß und nicht seinem persönlichen Rang entsprechend zu plazieren. Das Auswärtige Amt zeigte Verständnis dafür, doch angesichts antisemitischer Vorfälle in Deutschland Ende 1959 wurde der Zeitpunkt zur Umsetzung der arabischen Forderungen als ungeeignet erachtet, so daß Shinnar auch am Neujahrsempfang von 1960 noch als Botschafter ad personam teilnahm, wenn auch zum

53 Meroz an die Abteilung Westeuropa vom 8. 7. 1959, ISA, 301/5.

54 Zur sowjetischen Reaktion: Aryeh Lapid an den Generaldirektor vom 2. 7. 1959, ISA, PM 7224/54.

55 Niederschrift des Staatssekretärs über die Diplomatenkonferenz in Beirut vom 25.8. 1959, PA, 34b.

56 Bericht Shinnars vom 8. 1. 1954, ISA, 2413/7. 
letzten Mal. ${ }^{57}$ Später wurde sein persönlicher Rang den arabischen Forderungen entsprechend nicht mehr anerkannt, worauf Shinnar die diplomatischen Anlässe in Bonn künftig mied, zum Bedauern der deutschen Seite.

\section{Die Hakenkreuzschmierereien von 1960}

In Köln, dem Standort der Israel-Mission, existierte eine ziemlich große jüdische Gemeinde, die mit Unterstützung der öffentlichen Hand anstelle der in der „Kristallnacht" zerstörten alten Synagoge eine neues Gotteshaus baute. Das Gebäude wurde Ende 1959 fertiggestellt und in den ersten Tagen des Jahres 1960 mit Hakenkreuzen und antijüdischen Parolen beschmiert. Das war der Anfang einer Swastikawelle, die sich quer durch die Bundesrepublik zog, dann in ganz Europa ausbreitete und schließlich auch die Vereinigten Staaten erreichte. Die Drahtzieher, falls es sie überhaupt gab, wurden nie gefaßt. 58 Über die Hintergründe dieser antisemitischen Welle zirkulierten verschiedene Theorien. Die Polizei vermutete vor allem Jugendliche, Geistesgestörte und Rechtsextreme als Täter. Kühnere Theorien lasteten die antisemitische Welle der DDR und der Sowjetunion an. Andere beschuldigten wiederum die arabischen Staaten und die sich dort aufhaltenden NS-Flüchtlinge. Eine weitere Theorie vermutete sodann ein internationales nationalsozialistisches Komplott. Der Chef des israelischen Geheimdienstes „Mossad“, Iser Harel, wurde von Ministerpräsident Ben Gurion beauftragt, die Urheber der Welle aufzuspüren, doch seine Nachforschungen blieben erfolglos. ${ }^{59}$ Weit größere Bedeutung als der Urheberschaft wurde der Frage beigemessen, wie sich diese antisemitische Erscheinung so schnell ausbreiten konnte. Der Umstand, daß Hitlers Ideologie fünfzehn Jahre nach seinem Ende noch so stark unterstützt wurde, erfüllte die politische Führung der Bundesrepublik, führende Vertreter der jüdischen Gemeinschaft und Israels, aber auch die breite Öffentlichkeit mit Sorge. Die Bundesregierung war offensichtlich mit einem heiklen Problem konfrontiert, dem sie mit Aufklärungskampagnen in der Öffentlichkeit und in den Schulen entgegenzuwirken plante. Die Vertretungen im Ausland sollten zudem verstärkt auf die Aufrichtigkeit der westdeutschen Haltung und die antinazistische Gesinnung in der Bundesrepublik hinweisen. Der jüdischen Seite, einschließlich dem eigens von Adenauer aufgebotenen Goldmann, war in dieser Aufklärungskampagne eine wichtige Rolle zugedacht.

Die größte Protestveranstaltung gegen die antisemitische Welle fand im Februar 1960 auf dem Gelände des ehemaligen Konzentrationslagers Bergen-Belsen unter Beteiligung von Adenauer, Goldmann und Delegationen des Jüdischen Weltkongresses, Israels, der Vereinigten Staaten, Frankreichs, Deutschlands und weiterer Länder statt. ${ }^{60}$ Einige jüdische Persönlichkeiten kritisierten die Veranstaltung.

\footnotetext{
57 Meroz an Shinnar vom 21. 12. 1959, ISA, 582/1; Shinnar an die Abteilung Westeuropa vom 11. 1. 1960, ISA, 300/8.

58 Görschler/REINHARDT, Die Schande von Köln und Bonn; SCHÖNBACH, Reaktionen.

59 Tagebucheinträge vom 22. und 28. 1. 1960, BGA, BGD.

60 Goldmann an Blankenhorn vom 22.1. 1960, CZA, Z6/2034; Dr. Eleanor Sterling an
} 
Auf einer anderen Protestversammlung soll Adenauer vorgeschlagen haben, Jugendliche, die bei antijüdischen Schmierereien ertappt werden, „auf der Stelle zu verprügeln" ${ }^{61}$ Die Bestellung von hundertachtzigtausend Mörsergranaten aus Israel, eine freudige Überraschung für den jüdischen Staat, dürfte eine Geste des guten Willens angesichts des Swastikaskandals gewesen sein. ${ }^{2}$ Im Frühjahr 1960 stattete Altbundespräsident Heuss Israel einen privaten Besuch ab. Obwohl bereits seit längerem vorbereitet, wurde der Besuch als Botschaft interpretiert, daß Deutschland die NS-Verbrechen nicht vergessen habe. ${ }^{63}$

Die israelische Regierung reagierte auf die antisemitische Welle mit einer an die Bundesregierung gerichteten offiziellen Protestnote, die trotz fehlender diplomatischer Beziehungen protokollgemäß entgegengenommen wurde.64 Zahlreiche Stimmen - auch auf jüdischer Seite - plädierten für die Aufnahme diplomatischer Beziehungen zu Israel als besten Weg zur Beschwichtigung der jüdischen Gemeinschaft. Außerdem werteten sie einen solchen Schritt als eine Chance, die Entschlossenheit der Bundesregierung zu unterstreichen, solchen Vandalismus in Zukunft zu verhindern. Die antisemitische Welle hatte dem mit viel Mühe wiederhergestellten Ruf der Bundesrepublik schwer geschadet. ${ }^{65}$ Dennoch wies das Auswärtige Amt den Vorschlag entschieden zurück. Auch Golda Meir konnte sich mit ihm nicht anfreunden. In einem scharfen Brief verlangte sie von den ${ }_{n}$ Kollaborateuren" damit aufzuhören, „um Beziehungen zu betteln ${ }^{\text {“ }}{ }^{66}$ Der israelische Botschafter in Brüssel, Gideon Rafael, vertrat die gegenteilige Position: „Die antijüdischen und neonazistischen Erscheinungen haben dem internationalen Ansehen Deutschlands sehr geschadet, und alles deutet darauf hin, daß die Regierung Adenauer dafür sensibel ist. Diese Sensibilität ist angesichts der deutschen $\mathrm{Be}-$ fürchtung verständlich, die USA und England könnten sich auf der [für den Sommer 1960 geplanten] Gipfelkonferenz mit der UdSSR auf Kosten Deutschlands auf einen Kompromiß einigen [...] Auch wir sollten die Schwäche ausnutzen, in der sich die Regierung Adenauer zur Zeit befindet, um die Frage der Beziehungen in die richtige Bahn zu lenken. ${ }^{47}$ Doch Meir lehnte ab. Sie war nicht bereit, als Bittsteller aufzutreten. Es sei Sache der Deutschen, den ersten Schritt zu tun. Die Außenministerin wies Shinnar an, weder Verärgerung zu zeigen noch Drohungen auszusprechen, dafür aber Entschlossenheit zu demonstrieren und bei freundlich gesinnten Ministern für die israelischen Anliegen einzutreten. Der Ministerpräsident befürwortete Meirs Taktik. ${ }^{68}$

Zacharia Schuster vom 3. 2. 1960, YIVO, AJC, FAD-1, Box 26, Ger. West; MA'ARIV (Tel Aviv) vom 3. 2. 1960.

61 Frankfurter Allgemeine Zeitung vom 30. 1. 1960.

62 Shinnar an Fischer vom 20. 2. 1960, ISA, 301/15.

${ }^{63}$ Die britische Botschaft in Bonn an die Abteilung Levante vom 6. 1. 1960, PRO, FO 371/ 151170, VR 10318/1.

64 Shinnar an die Abteilung Westeuropa vom 11. 1. 1960, ISA, 300/8.

65 Von Brentano an Adenauer vom 18. 1. 1960, BArch, N 1239/158.

66 Rafael an Meir vom 26. 1. 1960, ISA, 301/19.

67 Ebd.

68 Savir an Fischer vom 4. 1. 1960, ISA, 3309/13. 
Auch die Bundesregierung hielt trotz der unangenehmen Situation, in der sie sich befand, entschlossen an ihrer Linie fest. Im April 1959 gab Adenauer Shinnar wieder einmal eine jener regelmäßigen Zusagen und bekräftigte sie im Januar 1960 erneut. ${ }^{69}$ Massiver Druck einzelner Bundesminister und der SPD sowie israelischer Stellen und jüdischer Vertreter aus westlichen Ländern ließen diesmal berechtigte Hoffnung auf eine endgültige Lösung der Beziehungsfrage aufkommen. Den Vorschlag Goldmanns, sich amerikanischen und französischen Drucks zu bedienen, wies Golda Meir jedoch entschieden zurück und versuchte, eine Beteiligung Goldmanns an der Angelegenheit zu unterbinden..$^{70} \mathrm{Ob}$ hier einmal mehr Meirs traditionelle Haltung zum Ausdruck kam oder ob die Rüstungsverhandlungen zwischen Peres und Strauß diesmal den Ausschlag gaben, bleibt dahingestellt. Sicher ist, daß die Verhandlungen die israelische Strategie bestärkten, wonach der Waffenkauf diplomatischen Beziehungen und anderen Formen des gegenseitigen Austausches vorzuziehen sei.

Fest steht auch, daß die Bundesrepublik damals nicht die geringste Absicht hatte, diplomatische Beziehungen zu Israel aufzunehmen. Adenauer, von Brentano und das Auswärtige Amt hatten die israelische Regierung unter allerlei Vorwänden irregeleitet. ${ }^{71}$ So wurde etwa die Angst vor Spannungen im Nahen Osten vorgeschoben oder die Notwendigkeit betont, vor der geplanten alliierten Gipfelkonferenz in Genf heikle Entscheidungen zu verschieben.72 Ein weiterer Vorwand bezog sich auf die mögliche Anerkennung der DDR durch arabische Staaten, und schließlich wurde auf den amerikanischen Wunsch hingewiesen, Spannungen im Vorfeld des Gipfels zu vermeiden. ${ }^{73}$ Adenauer soll Shinnar eine schriftliche Zusage hinsichtlich der Aufnahme diplomatischer Beziehungen angeboten haben, weigerte sich später jedoch, das Versprechen einzuhalten. Schließlich setzte der Bundeskanzler am 2. März 1960 einen Beschluß gegen die Aufnahme diplomatischer Beziehungen zu Israel durch. ${ }^{74}$ Der Versuch, die antijüdischen Schmierereien zur Aufnahme diplomatischer Beziehungen zu nutzen, war gescheitert.

\section{Waldorf Astoria}

Die beiden altgedienten Regierungschefs Adenauer und Ben Gurion trugen sich schon eine Weile mit dem Gedanken, eine persönliche Begegnung herbeizuführen, doch erst die Swastikawelle verhalf der Idee zum Durchbruch. Josephthal und Shinnar hatten für diese Eventualität bereits Vorbereitungen getroffen. Für Ben

69 Blankenhorn, Verständnis, S. 363-365.

70 Shinnar an die Abteilung Westeuropa vom 19. 2. 1960; Shinnar an Golda Meir vom 14. 2. 1960; Telegramm Shinnars an Yachil vom 16. 2. 1960; Yachil an Shinnar vom 16. 2. 1960, ISA, 301/18. Zur angeblichen amerikanischen und britischen Intervention zugunsten Israels: MA'ARIv (Tel Aviv) vom 24. 2. 1960.

71 Shinnar an Fischer vom 8. 2. 1960, ISA, 3309/25.

72 Ebd.

73 Telegramm Shinnars an Yachil und Fischer vom 4. 3. 1960, ISA, 301/17.

74 Ebd. 
Gurion bedeutete die direkte Begegnung eine Gelegenheit, aktuelle Fragen zu erörtern, und Adenauer erhoffte sich davon einen positiven Effekt für den angeschlagenen Ruf seines Staates. Anfang 1960 nahmen die Vorbereitungen für das Gipfeltreffen konkrete Gestalt an. ${ }^{75}$ Den Einträgen in Ben Gurions Tagebuch zufolge schlug Adenauer vor, das Treffen in Griechenland, entweder in Athen oder auf Rhodos abzuhalten. ${ }^{76}$ Am 9. Februar teilte Shinnar Golda Meir schriftlich mit, daß Adenauer angeboten habe, Ben Gurion in Israel zu treffen. ${ }^{77}$ Hierzu ist es jedoch nicht gekommen, da sich Adenauer und Ben Gurion im März 1960 zur gleichen Zeit in den USA aufhielten und daher ein Treffen in New York zustandekommen konnte. Zur Vorbereitung der Unterredung nahm Adenauer die guten Dienste seines Staatssekretärs Hans Globke in Anspruch. Globke war mit dem Geschäftsmann Julius Klein aus Chicago befreundet, ${ }^{78}$ der die beiden Regierungschefs persönlich kannte und offenbar im Hintergrund den Boden für das Gipfeltreffen bereitet hatte. Sowohl Ben Gurion als auch Adenauer haben Kleins Hilfe später gewürdigt, wovon etwa die Widmung für Klein auf einer Photographie der beiden Regierungschefs am Gipfel im Waldorf Astoria Hotel zeugt. ${ }^{79}$

Als Ort des Gipfeltreffens wurde das New Yorker Waldorf Astoria Hotel gewählt, wo sich beide Staatsgäste etwa zur gleichen Zeit aufhalten wollten. Die Assistenten beider Regierungschefs brauchten sich nur noch über den genauen Zeitpunkt zu verständigen. Shinnar machte Ben Gurion am 23. Februar 1960 mit den entsprechenden Einzelheiten vertraut. ${ }^{80} \mathrm{Am}$ 28. Februar informierte der Ministerpräsident Finanzminister Levi Eschkol über den Staatsbesuch in den Vereinigten Staaten und in Großbritannien sowie über das geplante Treffen zwischen Ben Gurion und Adenauer, das am 14. März 1960 im besagten Hotel in New York stattfand. Die israelische Flagge wurde nicht gezeigt, möglicherweise weil die Israelis Wert auf den vertraulichen Rahmen der Begegnung legten und vermeiden wollten, daß die israelische und die deutsche Flagge Seite an Seite im Wind wehten.

Am 5. Februar notierte Ben Gurion ein paar Gedanken in sein Tagebuch, die sich bei näherer Betrachtung als allgemeine Reflexionen über den bevorstehenden Gipfel, aber auch als konkrete Vorbereitung für die bevorstehenden Treffen mit Eisenhower, Adenauer und dem britischen Premierminister Harold Macmillan entpuppen. Ben Gurion reflektierte über den Sinn von Gipfelkonferenzen, das Ost-West-Verhältnis, über Israels Sicherheit sowie über die Beziehungen zur Sowjetunion, zu afrikanischen Staaten und zu Europa. All diese Themen kamen im Verlauf der erwähnten Begegnungen dann tatsächlich zur Sprache. Ben Gurion war also gut vorbereitet.

Das Treffen zwischen Ben Gurion und Adenauer fand auf Anregung Ben Gurions in der Hotelsuite des Bundeskanzlers statt. Die mit dem Altersvorsprung

\footnotetext{
75 Tagebucheinträge vom 7., 23. und 28. 2. 1960, BGA, BGD.

76 Tagebucheintrag vom 7. 2. 1960, BGA, BGD.

77 Shinnar an Meir vom 9. 2. 1960, ISA, 3309/13.

78 DoHRN, Globkes Verhältnis, S. 175.

79 Kopie im Besitz des Autors.

80 Tagebucheintrag vom 23. 2. 1960, BGA, BGD.
} 
Adenauers begründete Geste wurde von der israelischen Presse negativ aufgenommen. Adenauer sprach Deutsch, Ben Gurion Englisch. Beiden Staatsmännern standen Übersetzer zur Seite, die sie jedoch nicht in Anspruch nahmen. ${ }^{81}$

Adenauer war auf Ben Gurions Hilfe angewiesen, um Deutschlands guten Ruf wiederherzustellen. Er benötigte "moralische Güter", die sich als sehr kostspielig erwiesen. Ben Gurion verlangte dafür jedenfalls einen hohen Preis. Den Staatsbesuch in den Vereinigten Staaten hatte er mit einer riesigen „Einkaufsliste“ angetreten, in der Hoffnung, die Amerikaner zu einem Rüstungslieferanten des Staates Israel zu verwandeln, geradezu ein Alptraum für die US-Regierung. Ein amerikanisches Memorandum gibt Eisenhowers Standpunkt dazu wie folgt wieder: „Eisenhower glaubt aufrichtig, daß westeuropäische Nationen - Frankreich, Großbritannien und sogar Deutschland - zur Lieferung von Waffen an Israel besser geeignet wären als die Vereinigten Staaten. " $82 \mathrm{Da}$ Eisenhower über die westdeutschen Lieferungen an Israel informiert war, sind seine Worte wohl als Anspielung auf eine bereits bestehende Realität zu werten: Im Februar 1960 hatten Strauß und Peres in Paris ein umfangreiches Rüstungsabkommen vereinbart, das auch die Lieferung moderner Waffen einschloß und von Adenauer auf Ben Gurions Wunsch bestätigt wurde. ${ }^{83}$ Bestärkt von diesem Erfolg bemühte sich Ben Gurion bei der deutschen Seite anschließend um ein neues Kreditpaket, als die Schilumim bereits ihrem Ende zuneigten. Ben Gurion und sein engster Beraterkreis beschlossen, eine Anleihe für die Entwicklung der Negev-Wüste aufgeteilt in zehn Jahresraten zu beantragen. Ben Gurions Zukunftsvisionen konzentrierten sich vor allem auf dieses Gebiet, das mehr als $60 \%$ der Landesfläche bedeckte.

In der Unterredung mit dem Bundeskanzler verlangte Ben Gurion 500 Millionen Dollar. Adenauer mag mit einem finanziellen Gesuch gerechnet haben. Doch seine fachliche Kompetenz in Wirtschaftsfragen war nicht die beste. Gleichwohl gab er ohne lange zu zögern Ben Gurion eine positive Antwort, vermutlich um ihn nicht vor den Kopf zu stoßen. Von Eckardt erinnert sich an folgende Szene: „Ich sehe es noch vor mir, wie Adenauer eine Hand auf den Arm Ben Gurions legte und sagte: ,Seien Sie sicher, wir werden Israel nicht im Stich lassen'. "84 Die Antwort des Bundeskanzlers war zweifellos aufrichtig. Doch er hatte keine Summe genannt und war sich sicher auch bewußt, daß so eine Anleihe die Zustimmung des Bundestages erforderte.

Ganz anders interpretierten die Israelis Adenauers Antwort. Geblendet vom vermeintlichen Erfolg beschlossen sie, den Kanzler beim Wort zu nehmen. Obwohl vereinbart war, das Versprechen vertraulich zu behandeln und Shinnar mit Adenauers Hilfe die Ausarbeitung der Einzelheiten zu übertragen, wurde die Angelegenheit in Israel schon am nächsten Tag der Presse zugespielt. Ben Gurion

81 JELINEK/Blasius, Ben Gurion und Adenauer, S. 309-344.

82 Memorandum über das Gespräch Präsident Eisenhowers mit Premierminister Ben Gurion vom 10.3. 1960, Eisenhower Library, Abilene, Kansas, White House Office, Office of the Staff, International Series: Israel (2), (March-August 1960).

83 GôlaN, Shimon Peres, S. 117; Aufzeichnung vom 2. 2. 1960, PA, 84.00-84-20/2, 92.19; Tagebucheintrag Ben Gurions vom 18. 2. 1960, BGA, BGD.

${ }^{84}$ ECKARDT, Unordentliches Leben, S. 610. 
rechtfertigte sich später damit, er habe seine Einwilligung zur Veröffentlichung in einem Zustand der Erschöpfung gegeben, doch man kann sich des Eindrucks nicht erwehren, daß der israelische Premierminister und sein engster Beraterkreis die Bundesrepublik damit vor vollendete Tatsachen stellen wollten. ${ }^{85}$

Bonn dementierte umgehend, doch die arabischen Proteste, die man offensichtlich vermeiden wollte, brachen mit voller Kraft auf Bonn herein und gefährdeten die Einlösung von Adenauers Versprechen. Das Auswärtige Amt versuchte die Wahrheit über das Treffen im Hotel Waldorf Astoria herauszufinden und tat alles in seiner Macht stehende, um die Staaten des Nahen Ostens zu beschwichtigen. Ben Gurions vorschneller Gang zur Presse könnte außerdem bezweckt haben, die erhebliche Kritik im eigenen Land zum Schweigen zu bringen. Der israelische Ministerpräsident hatte im voraus mit solcher Kritik gerechnet und wollte der Öffentlichkeit die Erfolge seiner Politik präsentieren, ohne auf die vertraulichen Rüstungsgeschäfte einzugehen. Die Presse und die Öffentlichkeit in Westdeutschland begrüßten Adenauers Schritte. ${ }^{86}$ Sie wurden als Maßnahmen zur Verbesserung des internationalen Ansehens der Bundesrepublik nach den antijüdischen Schmierereien verstanden. ${ }^{87}$

Des Kanzlers Umgebung witterte den Konflikt über die Art der Finanzhilfe an Israel und deren Höhe sofort und drängte auf eine möglichst unverbindliche Wiedergabe seiner Zusage in einer amtlichen Presseerklärung: Die Bundesrepublik „werde Israels Entwicklung weiter mit Aufmerksamkeit verfolgen“, hieß es in Bonn, während die Israelis darauf beharrten, Adenauer habe gesagt, „die Bundesrepublik werde Israel weiter unterstützen“. Der Bundeskanzler bestätigte auf Anfrage, das Wort „Unterstützung " verwendet zu haben. ${ }^{88}$ Abgesehen davon kam es zu Meinungsverschiedenheiten über die Höhe der Summe und die Aufteilung in Jahresraten. Die deutsche Seite, der Bundeskanzler mit eingeschlossen, bestritt, eine feste Summe versprochen zu haben, während die Israelis sich hartnäckig an eine positive Antwort Adenauers auf Ben Gurions Gesuch von 400-500 Millionen Dollar in zehn Jahresraten erinnerten. Der Kredit mit dem Kodenamen "Geschäftsfreund" erforderte langwierige Verhandlungen, die erst 1966 zum Abschluß kamen. Die Israelis verzichteten schließlich auf die ursprünglichen Forderungen vom Waldorf Astoria-Gipfel und erhielten dafür Finanzhilfe auf jährlicher Basis. ${ }^{89}$

Ein weiterer nicht zu vernachlässigender Aspekt der Begegnung zwischen Bundeskanzler Konrad Adenauer und Ministerpräsident David Ben Gurion betrifft die persönliche Ebene. Den Dokumenten und persönlichen Aufzeichnungen zufolge war das Verhältnis zwischen den beiden Regierungschefs schon vor der persönlichen Begegnung von großem gegenseitigen Respekt geprägt. Im Gespräch

85 Der Generaldirektor an Shinnar vom 29. 3. 1960, ISA, 3294/4.

86 FAZ vom 16. 3. 1960, Die WeLT vom 16. 3. 1960, STUTTGARTER ZEITUNG vom 17. 3. 1960.

87 STUTTGARTER ZEITUNG vom 17. 3. 1960; DIE WELT vom 25. 3. 1960; NBC, Meet the Press vom 20.3. 1960.

88 Der Minister Plenipotentiary in Washington an den Generaldirektor vom 18. 3. 1960, ISA, 3294/4.

89 Jelinek/Blasius, Ben Gurion und Adenauer, S. 309-344; Der DeUTSCH-ISRAELISCHE Dialog, Bd. 1, S. 149-157. 
selbst fanden sie - wie Adenauer in seinen Memoiren schreibt - „sogleich Kontakt zueinander". .90 Diese Aussage wird durch andere spätere Ausführungen und Schilderungen ebenso bestätigt wie durch Photographien der Begegnung. Die beiden Staatsmänner wollten sich kennenlernen und waren froh über die sich ihnen bietende Gelegenheit. Einzig Chaim Yachil, damals Generaldirektor des israelischen Außenministeriums, beurteilte die Begegnung nachträglich eher negativ: „Nach dem Protokoll zu urteilen - ich kann nur hoffen, daß das Gespräch darin nicht genau wiedergegeben ist - scheint mir dieses Treffen eines der schwächsten Glieder im großen Unternehmen des Premierministers in den USA und in England gewesen zu sein. Ich konnte nicht den Eindruck gewinnen, daß die beiden Persönlichkeiten bei ihrer ersten Begegnung eine gemeinsame Sprache und Gesprächsbasis finden konnten, obwohl Adenauer die Anliegen des Premierministers im konkreten Bereich vorbehaltlos guthieß."

Ein Thema, dessen Behandlung sowohl von der Presse als auch von den involvierten Personen erwartet wurde, kam nicht zur Sprache: die Aufnahme diplomatischer Beziehungen. $\mathrm{Ob}$ es im voraus so vereinbart war, wie zumindest eine Zeitung behauptete, oder ob Ben Gurion aus eigener Initiative darauf verzichtete, ist schwer zu sagen. ${ }^{92}$ Die Zeitung Ma'ariv meinte, Ben Gurion werde das Thema nicht erwähnen, solange keine deutsche Initiative vorliege, was der etablierten Linie der israelischen Politik entsprach. In Anbetracht von Ben Gurions Haltung und der relativ großen Bedeutung, die er diplomatischen Beziehungen einräumte, darf davon ausgegangen werden, daß die Ausklammerung des Themas auf einen persönlichen Entschluß des israelischen Staatschefs zurückging. Ben Gurion sah sich veranlaßt, auf einen Eckpunkt des Dreiecks Wirtschafts-/Finanzhilfe, diplomatische Beziehungen und Militärhilfe zu verzichten, um die anderen zwei Eckpunkte zu fördern. Der israelische Regierungschef hat das Spiel nicht nur durchschaut. Er hat es selbst erfunden. Die diplomatischen Beziehungen vernachlässigte er bewußt, was von Adenauer als taktvolles Verhalten interpretiert und positiv vermerkt wurde. Der Kanzler versprach darauf Unterstützung bei den zwei verbleibenden Bitten, die Ben Gurion besonders am Herzen lagen. Da die Begegnung die von Adenauer erhoffte Publizität erreichte, dürften beide Seiten zufrieden gewesen sein. Als der Bundeskanzler von der Presse in Washington auf den Antisemitismus in der Bundesrepublik angesprochen wurde, streckte er den Journalisten ein gemeinsames Foto mit Ben Gurion entgegen.93

Die Begegnung im Hotel Waldorf Astoria im Jahre 1960 war das spektakulärste Ereignis im deutsch-israelischen Verhältnis seit der Unterzeichnung des Luxemburger Abkommens im September 1952. Im selben Jahr sollte noch ein weiteres dramatisches Ereignis folgen, das zwar ganz anders beschaffen, aber nicht weniger bahnbrechend war für den Dialog der beiden nach dem Holocaust.

\footnotetext{
90 AdENAUER, Erinnerungen 1954-1963, S. 32.

91 Yachil an Shinnar vom 29. 3. 1960, ISA, 3294/4.

92 MA'ARIV (Tel Aviv) vom 14. 3. 1960.

93 NBC, Meet the Press, Nr. 12, Bd. 4 vom 20. 3. 1960.
} 
